\title{
Prognostic factors and survival after complete resection of pulmonary metastases from colorectal carcinoma: Experiences in 167 patients
}

\author{
Joachim Pfannschmidt, MD \\ Thomas Muley, PhD \\ Hans Hoffmann, MD, PhD \\ Hendrik Dienemann, MD, PhD
}

From the Department of Thoracic Surgery,
Thoraxklinik Heidelberg, University of
Heidelberg, Heidelberg, Germany.
Received for publication Dec 18, 2002; re-
visions requested Jan 22, 2003; revisions
received Feb 22, 2003; accepted for publi-
cation March 18, 2003.

Address for reprints: Joachim Pfannschmidt MD, Department of Thoracic Surgery, Thoraxklinik Heidelberg, Amalienstr. 5, D-69126, Heidelberg, Germany (E-mail: joachim. pfannschmidt@ thoraxklinik-heidelberg.de).

J Thorac Cardiovasc Surg 2003;126:732-9

Copyright $\odot 2003$ by The American Association for Thoracic Surgery

$0022-5223 / 2003 \$ 30.00+0$

doi:10.1016/S0022-5223(03)00587-7
Objective: Surgical resection is an important form of treatment for pulmonary metastases from colorectal carcinoma. We analyzed the clinical course, outcome, and prognostic factors after surgery.

Methods: Between 1985 and 2000, 167 patients (103 men, 64 women) underwent complete pulmonary resection of metastatic colorectal carcinoma. Only patients who met the criteria for potentially curative operation, in particular, control of the primary tumor, ability to resect all metastatic disease, and no other extrapulmonary metastases, were included.

Results: The overall 5-year survival was $32.4 \%$. A significantly longer survival was observed in multivariate analysis in patients without lymph node involvement compared with patients with pulmonary or mediastinal lymph node metastases or both. The number of pulmonary metastases significantly influenced survival. In patients with a solitary metastasis, we observed a 5-year survival of $45 \%$, whereas the rate was $19.8 \%$ in patients with more than a single metastasis. In multivariate analysis, we also found the prethoracotomy carcinoembryonic antigen serum level to be an independent significant prognostic factor for survival. In patients with a serum carcinoembryonic antigen level exceeding $5 \mathrm{ng} / \mathrm{mL}$ and in patients with a serum carcinoembryonic antigen level in the normal range, the 5-year survivals were $22.7 \%$ and $48.3 \%$, respectively.

Conclusions: We conclude that pulmonary resection of metastatic colorectal carcinoma is safe and results in long-term survival. Thoracic lymph node metastases, serum carcinoembryonic antigen level before metastasectomy, and the number of pulmonary metastases were identified as prognosis-related criteria for surgery.

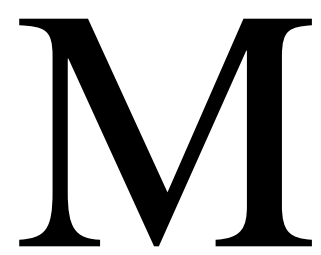

ore than half of the patients undergoing resection for colorectal cancer can be expected to have recurrence of the disease. $^{1,2}$ The most frequent sites of recurrence are the liver and the lungs. Patients with untreated metastatic disease have a median survival of less than 10 months and a 5-year survival of less than $5 \% .^{3}$ Conventional treatment of stage IV colorectal carcinoma in most patients is palliative. Fluorouracil-based chemotherapy is considered to be the standard treatment but seldom produces long-term survival.

The first resection of a pulmonary metastasis was performed by Divis in $1927 .^{4}$ Since then, surgery remains the only effective treatment for patients with isolated pulmonary metastases. Several retrospective studies have shown that patients with isolated pulmonary metastases benefit from resection. The published 5-year survivals after pulmonary metastasectomy of colorectal origin range from $21 \%$ to $62 \%$. $^{5-11}$ Resection of hepatic and pulmonary metastases, secondary to colorectal cancer, is 
also regarded as safe and results in long-term survival. ${ }^{12}$ However, reliable simple prognostic factors are still in great demand. This article focuses on the outcome, long-term results, and factors associated with prolonged survival in a relatively large series of patients who underwent curative resection for metastatic colorectal cancer of the lungs.

\section{Patients and Methods}

Between January 1985 and January 2000, 167 patients underwent complete resection of metastatic colorectal carcinoma at our institution. Table 1 is a summary of pertinent patient demographics. In all patients, the primary tumor was treated by curative resection. Tumor-node-metastasis classification revealed a $\mathrm{T}$ stage less than 4. Patients with a history of colorectal carcinoma and 1 or more pulmonary nodules were evaluated by physical examination, chest $\mathrm{x}$-ray films, chest computed tomographic (CT) scans, ultrasonography of the abdomen, and bone scans. In cases of uncertain clinical or radiologic findings, further examinations were performed to exclude extrapulmonary metastases. The primary site was confirmed to be controlled by an abdominal CT scan or colonofiberscopy.

Only patients who met the criteria for potentially curative operation were included. The preconditions valid for potentially curative operation were as follows: (1) The metastases seem to be technically resectable, (2) the general and functional risks are tolerable, (3) the primary tumor is controlled, and (4) no extrathoracic lesions are detected (with the exception of hepatic lesions, in which it is possible to completely remove both hepatic and pulmonary metastases).

Cases were included only if the histologic features of the pulmonary metastases were compatible with the histology of the primary lesion and characteristic for pulmonary metastases of colorectal origin.

All patients were retrospectively analyzed for age and sex, primary tumor location, number and type of pulmonary resections, completeness of resection, number of pulmonary metastases, disease-free interval (DFI), infiltration of pulmonary or mediastinal lymph nodes, prethoracotomy carcinoembryonic antigen (CEA) serum level, 30-day mortality, and long-term survival. The end point for the assessment of efficiency was death.

In total, 215 thoracic procedures were performed in 167 patients who underwent curative resection. The surgical approach was chosen according to the location and number of pulmonary nodules. The resection of peripherally located nodules was performed by sternotomy or, in case of bilateral metastases, by bilateral sequential approach (Table 1). The type of lung resection for metastasectomy ranged in most patients from wedge resection to various types of anatomic lung resections in selected cases (Table 1). When in doubt of the histology between the primary bronchial adenocarcinoma and metastases of colorectal origin in frozen sections, we then performed a lobectomy if the patient was an operable candidate for lobectomy. In 30 patients, we performed a lobectomy of a solitary lung mass that was subsequently determined to be a metastasis. Systematic hilar and mediastinal lymph node dissection were performed concurrently with all procedures. Systematic lymph node dissection includes 4 compartments in the right-sided thoracotomy (paratracheal, infracarinal, inferior mediastinal, and hilar) and 4 compartments in the left (aortic, infracari-

\section{TABLE 1. Patients' characteristics}

\begin{tabular}{lc}
\hline Age & 60.2 y (25-81 y) \\
Mean (range) & 103 \\
Sex & 64 \\
Male & Patients (No.) \\
Female & \\
Approach for patients with first-time & 19 \\
$\quad$ operation & 85 \\
Bilateral/staged & 57 \\
Unilateral & 4 \\
Sternotomy & 2 \\
Clamshell & Procedures (n $=215)$ \\
Video-assisted thoracic surgery & 99 \\
Types of pulmonary resection & 42 \\
Wedge resection & 69 \\
Segmental resection & 5 \\
Lobectomy/bilobectomy & \\
Pneumonectomy & \\
\hline
\end{tabular}

nal, inferior mediastinal, and hilar). A total of 186 thoracotomies were performed as initial surgical resection in patients with lung metastases of colorectal carcinomas compared with 29 thoracotomies in patients with recurrent pulmonary metastases. These 29 repeat resections were performed in 24 of the 167 patients who presented with recurrent pulmonary metastases after complete resection.

\section{Statistical Analysis}

The data were analyzed by SPSS for Windows software (Statistical Package for Social Science, SPSS Inc, Chicago, Ill).

Critical values of prognostic factors differentiating between patient groups with good and poor prognosis were determined by the crit level procedure described by Abel and colleagues ${ }^{13}$ The best cutoff value was defined as the value that best discriminated between good and poor prognosis. Probability of survival was then analyzed by the Kaplan-Meier method ${ }^{14}$ using the date of pulmonary resection as the starting point. For patients who underwent bilateral sequential metastasectomy, the date of the second operation was used as the starting point. The significance of differences between subgroups was calculated using the log-rank test. ${ }^{15}$ The relationship between the single parameters in the test groups was calculated by the Fisher exact test. ${ }^{16}$ For the multivariate analysis of prognostic factors, the Cox model ${ }^{17}$ was used. Factors analyzed included age, sex, localization of the primary tumor, lymph node metastases, prethoracotomy serum CEA level, number of metastases, DFI, surgery for hepatic metastases, and repeated surgery.

\section{Results}

The 30 -day mortality rate was $1.8 \%$ (3/167 patients). There were 2 postoperative deaths caused by septic multiorgan failure after postoperative pneumonia. One patient postoperatively developed sudden cardiac failure. The mean follow-up period of surviving patients (censored patients) was $58.6 \pm 42.7$ months (0.5-183.9 months). The cumulative 5 -year survival of all patients was $32.4 \%$, and the median survival was 40.2 months (Figure 1). Of the prognostic factors analyzed, age, sex, localization of the primary tu- 


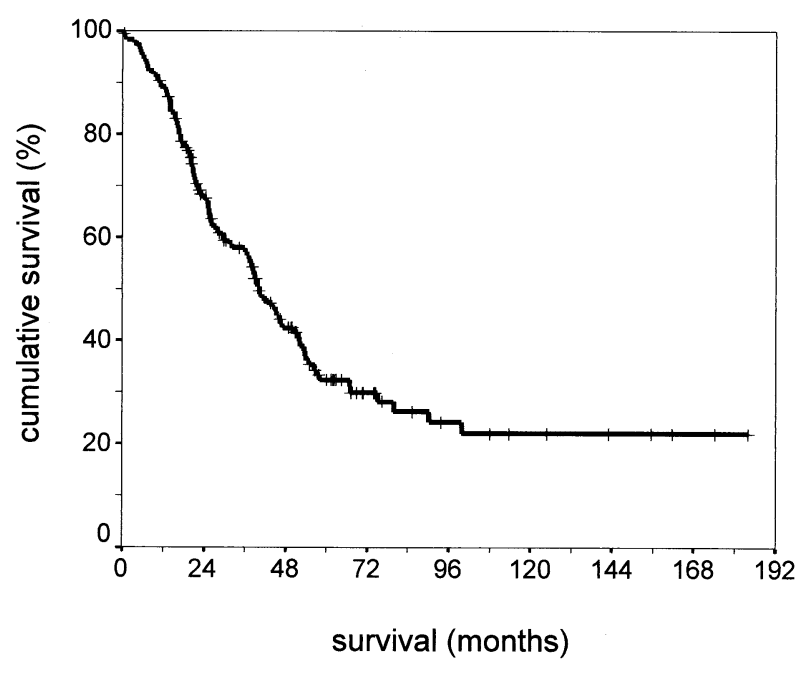

Figure 1. Probability of survival (death from any cause) of $\mathbf{1 6 7}$ patients after curative pulmonary resection of metastatic colorectal carcinoma. Zero time on the abscisse represents the date of pulmonary resection.

mor, type of resection, DFI, complete resection of hepatic metastases, and repeated thoracotomy did not significantly influence survival. No significant difference in long-term survival was found in patients after lobectomy of a single lung metastasis compared with patients after wedge resection $(P=.424)$. However, the number of metastases, lymph node involvement, and prethoracotomy CEA serum levels exceeding $5 \mathrm{ng} / \mathrm{mL}$ were found to be independent prognostic factors for survival after metastasectomy (Table 2).

No significant difference in long-term survival was detected in patients after complete resection of lung metastases from rectal carcinomas compared with patients with lung metastases from colon cancer (Table 2).

Pulmonary and mediastinal lymph node metastases were found in 32 patients, whereas 135 patients demonstrated no evidence of lymph node metastases. The nodal status significantly influenced survival. The median survival time was 20.2 months in patients with pulmonary or mediastinal lymph node metastases or both compared with 47.1 months in patients without lymph node involvement (Table 2, Figure 2). In patients with a single pulmonary metastasis, lymph node involvement was found in 15 patients, whereas 69 patients have had no evidence of lymph node metastases.

CEA levels before surgical treatment were documented in the records of 104 patients. In 63 patients, no serum CEA data were available. Univariate analysis showed a significantly poorer overall survival in patients with a serum CEA level exceeding $5 \mathrm{ng} / \mathrm{mL}$ compared with patients with a serum CEA level in the normal range (Table 2, Figure 3).

The number of pulmonary metastases ranged from 1 to 35 , with a median of 1 metastasis. In 2 patients, a diffuse metastatic infiltration of the resected lobe was found. The number of pulmonary metastases significantly influenced survival. The cutoff value for the number of pulmonary metastases that differentiated best between patients with good and poor prognosis was found to be 1 pulmonary metastasis (Table 2, Figure 4). However, patients with up to 4 lung metastases (139/167) showed a significantly better overall survival compared with patients with more than 4 metastatic lesions (median: 42.2 months vs 26.1 months, $P$ $=.022)$.

Pulmonary metastases were synchronously diagnosed in 22 patients; 145 cases presented a metachronous disease. The 5-year survival time in patients with synchronously diagnosed pulmonary metastases was $38.8 \%$ (median: 36.6 months) and $41.3 \%$ (median: 40.3 months) in patients with metachronous disease and did not differ significantly in both groups $(P=.93)$. The median DFI between the resection of the primary colorectal tumor and the detection of pulmonary metastases was 29.0 months (range; -2.1 months-335.6 months, Table 3). By our methods, we could not find a significant cutoff value for DFI that would differentiate between patients with good and poor prognosis (Table 2).

The complete prior resection of hepatic metastases showed no significant influence on survival (Table 2). Of the 20 patients who underwent resection of liver metastases, 8 and 12 patients showed synchronous and metachronous disease, respectively. The median survival time for patients with synchronous hepatic metastases was 16.1 months versus 39.8 months for patients with metachronous metastases. In the univariate analysis, no significant difference was shown between the subgroups $(P=.284)$.

In 24 patients, repeat thoracotomies (1-3) were performed for recurrent lung metastases after the initial complete resection of pulmonary metastases. Repeat thoracotomy for recurrent lung metastases was not associated with an increase in morbidity or mortality or with a significant difference in survival (Table 2).

For multivariate analysis, 2 different models were applied. In a first model, including all patients, the number of lung metastases $(1 \mathrm{vs}>1)$ and lymph node involvement (metastatic vs tumor-free) were found to be independent prognostic factors $(P=.0059$ and $P<.00001$, respectively). In a second model, the serum CEA value ( $\leq 5 \mathrm{ng} / \mathrm{mL}$ vs $>5 \mathrm{ng} / \mathrm{mL}$ ) was included. Therefore, the statistical power was less informative, because only 104 patients with available CEA values could be included. However, in the latter model, serum CEA $(P=.037)$ and lymph node involvement $(P=.042)$ retained significance as independent prognostic factors, whereas the number of metastases showed a reduced, nonsignificant discriminatory power $(P=.059)$ compared with the results found within the complete study population (Table 4). 
TABLE 2. Survival and potential prognostic factors tested for univariate analysis

\begin{tabular}{|c|c|c|c|c|c|}
\hline & $\mathbf{N}$ & $\begin{array}{c}\text { 5-year-survival } \\
(\%)\end{array}$ & $\begin{array}{l}\text { Median survival } \\
\text { (mo) }\end{array}$ & $\begin{array}{c}95 \% \mathrm{Cl} \\
(\mathrm{mo})\end{array}$ & $P$ value \\
\hline Overall & 167 & 32.4 & 40.2 & $34.2-46.2$ & \\
\hline \multicolumn{6}{|l|}{ Gender } \\
\hline Male & 103 & 32.6 & 39.6 & $31.7-47.6$ & \\
\hline Female & 64 & 32.3 & 40.3 & $30.8-49.9$ & .98 \\
\hline \multicolumn{6}{|l|}{ Histology } \\
\hline Colon & 93 & 34.5 & 43.9 & $29.9-58.0$ & \\
\hline Rectum & 74 & 30.2 & 39.8 & $34.9-44.8$ & .68 \\
\hline \multicolumn{6}{|l|}{ Lymph node involvement } \\
\hline None & 135 & 38.7 & 47.1 & $37.0-57.1$ & \\
\hline Lobar. hilar & 16 & n.d. & 21.5 & $14.4-28.6$ & .014 \\
\hline Mediastinal & 16 & 0 & 15.3 & $12.1-18.6$ & .0227 \\
\hline \multicolumn{6}{|l|}{ Number } \\
\hline 1 & 84 & 45.0 & 53.5 & $40.9-66.2$ & \\
\hline$>1$ & 83 & 19.8 & 28.0 & $16.9-39.1$ & .0013 \\
\hline \multicolumn{6}{|l|}{ CEA } \\
\hline $5 \leq$ & 52 & 48.3 & 57.9 & $39.7-76.1$ & \\
\hline$>5 \mathrm{ng} / \mathrm{mL}$ & 52 & 22.7 & 29.7 & $16.6-42.7$ & .0057 \\
\hline \multicolumn{6}{|l|}{ OP liver metastases } \\
\hline None & 147 & 32.4 & 42.2 & $34.9-49.6$ & \\
\hline OP & 20 & 33.0 & 38.0 & $0.0-79.5$ & .39 \\
\hline \multicolumn{6}{|l|}{ DFI } \\
\hline-6 & 22 & 38.8 & 36.6 & $28.8-44.4$ & \\
\hline $6-12$ & 14 & 40.0 & 38.2 & $5.4-71.0$ & \\
\hline $12-24$ & 37 & 28.4 & 38.8 & $9.0-68.6$ & \\
\hline$>24$ & 94 & 30.7 & 42.2 & $34.1-50.4$ & .98 \\
\hline \multicolumn{6}{|l|}{ OP for recurrent metastases } \\
\hline None & 143 & 35.4 & 39.6 & $29.8-49.5$ & \\
\hline $\mathrm{OP} \rightarrow$ complete resection & 17 & 24.5 & 52.0 & $36.0-67.9$ & .95 \\
\hline $\mathrm{OP} \rightarrow$ incomplete resection & 7 & 14.3 & 38.0 & $6.7-69.3$ & .17 \\
\hline
\end{tabular}

\section{Discussion}

The present study was conducted to describe criteria for selecting patients with pulmonary metastases in an attempt to identify patients who would benefit from surgical resection. A complete resection was performed in all cases, and the 5-year probability of survival was $32.4 \%$. In a multivariate analysis of a series of 215 thoracic surgical procedures in 167 patients, the results indicate that the presence or absence of thoracic lymph node metastases and the prethoracotomy CEA serum level are independent, significant prognostic factors for survival after metastasectomy. In the univariate analysis, the number of pulmonary metastases was a significant variable. Several studies concerning surgery for pulmonary metastases from colorectal carcinoma have been published, and the reported 5-year survivals ranged from $21 \%$ to $62 \% .^{5-11,18}$ In the present study, our data for survival after complete resection are consistent with the survival data presented in 4 large series that specifically addressed the issue of long-term outcome after surgery for pulmonary metastases from colorectal cancer. Okumura and colleagues ${ }^{6}$ reported from a series of 159 patients (5 incomplete resected cases), Saito and colleagues ${ }^{19}$ reported from a series of 165 patients, McAfee and colleagues ${ }^{9}$ reported from a series of 139 patients, and McCormack and colleagues $^{2}$ reported from a series of 144 patients with a 5-year survival of $40.5 \%, 39.6 \%, 30.5 \%$, and $40 \%$, respectively.

Age, sex, or location of the primary carcinoma (rectum vs colon) have, so far, never been reported as significant prognostic factors of survival. ${ }^{9,18-20}$ Surgical procedures for resection in our study included unilateral thoracotomy, staged bilateral thoracotomy, and median sternotomy. When all metastases were resected, the method chosen did not influence long-term survival in our series. In contrast with our finding, Saito and colleagues ${ }^{19}$ reported a significantly lower survival for simultaneous operations versus a sequential mode of operation. In our study, we found no significant difference in long-term survival after lobectomy compared with wedge resection even in patients with a single lung metastasis.

Median sternotomy was considered the procedure of choice for the initial exploration in the first half of our study interval. Recently, in the era of high-resolution CT, median sternotomy was reserved for patients with bilateral peripherally located nodules. However, we, among others, ${ }^{21}$ be- 


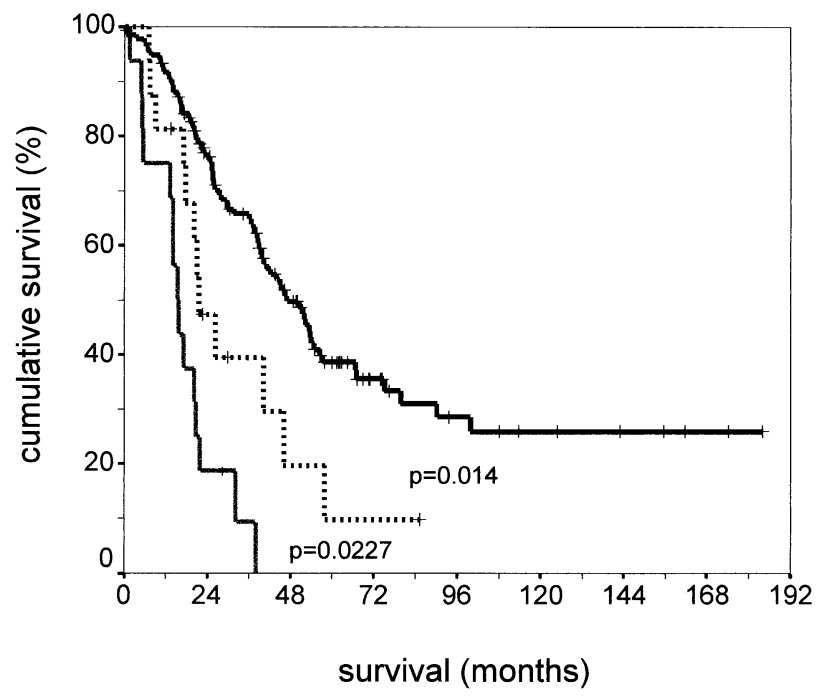

Figure 2. Probability of survival (death from any cause) of patients with lymph node involvement compared with patients with no lymph node involvement. Zero time on the abscisse represents the date of pulmonary resection (log-rank test). Patients with no lymph node involvement tended to survive longer than those with metastatic lobar or hilar lymph nodes ( $P=.014)$. Patients with metastatic lobar or hilar lymph nodes had a survival advantage over patients with positive mediastinal lymph nodes $(P=.023)$. No lymph nodes involved, $\mathbf{n}=135$ (black solid line); metastatic lobar or hilar lymph nodes, $\mathbf{n}=16$ (black dotted line); metastatic mediastinal lymph nodes, $\mathbf{n}=16$ (gray solid line).

lieve that open thoracotomy, with thorough palpation of the inflated and deflated lung, remains mandatory even in the light of modern CT sensitivity. The video-assisted approach is only of diagnostic value. ${ }^{22}$

In our study, lung resections were performed by removing pulmonary metastases with the most limited parenchymal resection permitting a curative resection. ${ }^{23}$ Surgical resection by means of wedge resection, segmentectomy, and, in selected cases, lobectomy or pneumonectomy, was completed by a systematic interlobar, hilar, and mediastinal lymph node dissection.

Mediastinal and hilar lymph node involvement is rarely examined in the literature. Lymph node metastases in cases of pulmonary metastases from colorectal carcinoma are being reported. ${ }^{6,18,24-27}$ In our study, 32 patients were diagnosed with pulmonary or mediastinal lymph node metastases or both after performing lymph node dissection. Multivariate analysis showed a statistically significant difference in survival between patients with lymph node involvement and those without lymph node metastases. The median survival time was also significantly longer for the subgroup of patients with only pulmonary lymph node involvement versus the group of patients with pulmonary and mediastinal lymph node metastases. Suggesting that metastases them-

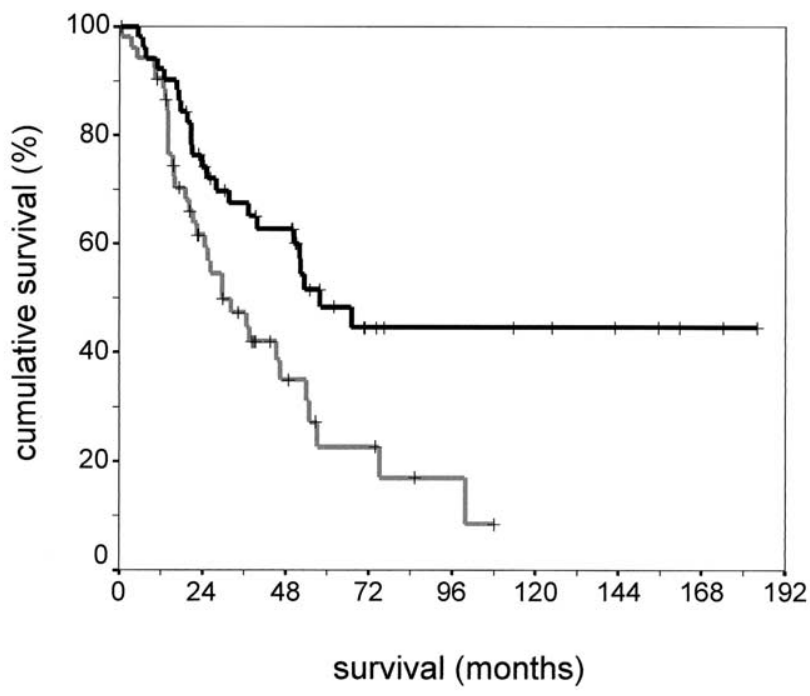

Figure 3. Probability of survival (death from any cause) of patients with elevated preoperative carcinoembryonic antigen (CEA) serum level versus patients with normal preoperative CEA serum level $(P=.0057)$. Zero time on the abscissa represents the date of pulmonary resection (log-rank test). CEA $5 \mathrm{ng} / \mathrm{mL}$ or less, $\mathrm{n}=52$ (black solid line); CEA more than $5 \mathrm{ng} / \mathrm{mL}, \mathrm{n}=52$ (gray solid line).

selves induce metastases remains intriguing. Thus, the belief that systematic mediastinal and hilar lymph node dissection is important offers a further understanding in pulmonary metastases and remains a significant prognostic factor. This may have an impact on further adjuvant treatment regimen. Consequently, any procedure sparing lymph node dissection should be regarded as incomplete. In 15 of 84 patients with a single metastasis, lymph node involvement was detected by systematic lymph node dissection. Thus, pulmonary resection of a single nodule by videoassisted endoscopic surgery without systematic mediastinal and hilar lymph node dissection is not recommended.

In assessing the mediastinal lymph nodes for the possible presence of metastatic tumor, a positive CT result (lymph nodes $>1.0 \mathrm{~cm}$ ) was confirmed in 7 of 16 patients by positive histologic evaluation (false negative rate: $56 \%$ ). In our current approach, the histologic evaluation of enlarged mediastinal lymph nodes must always be performed, because the finding of metastatic nodal involvement would determine inoperability or indicate a systemic chemotherapy. In a patient with a history of colorectal cancer and multiple pulmonary nodules and significant mediastinal lymph node enlargement detected in the CT scan, we perform a mediastinoscopy (or other invasive diagnostics, eg, transbronchial needle aspiration). In case of positive nodes, we do not suggest pulmonary metastasectomy. If we detect positive lymph nodes during sequential metastasectomy on 


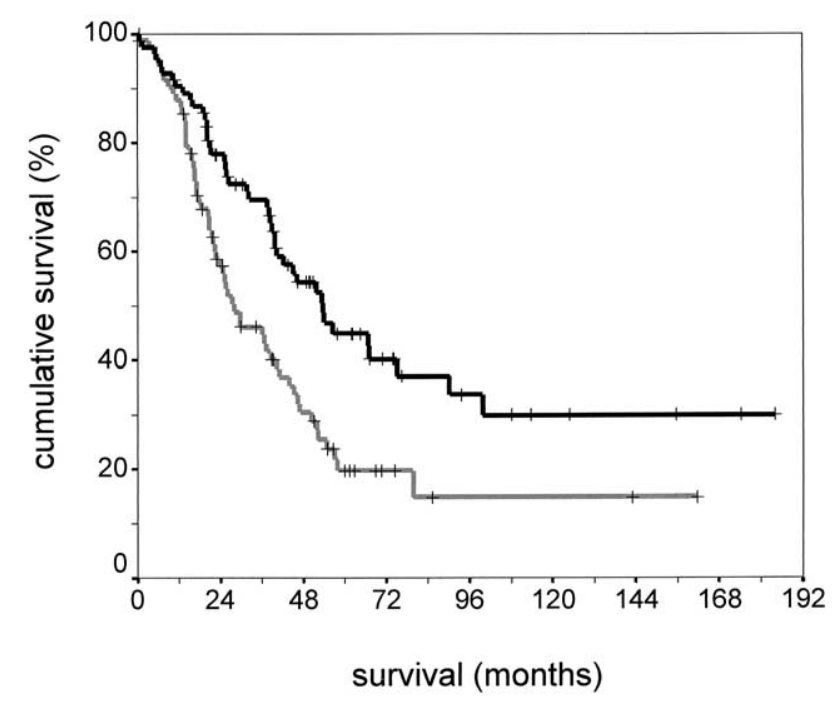

Figure 4. Probability of survival (death from any cause) of patients with a single pulmonary metastasis versus more than 1 metastasis $(P=.0013)$. Zero time on the abscissa represents the date of pulmonary resection (log-rank test). One metastasis, $\mathbf{n}=$ 84 (black solid line); more than 1 metastasis, $n=83$ (gray solid line).

1 side, we postpone the contralateral operation and suggest a systemic chemotherapy.

Recently, several reports have revealed that an elevated prethoracotomy serum CEA level showed a significant adverse effect on survival. ${ }^{8,9}$

Multivariate analysis in our series also revealed the prethoracotomy CEA serum level to be an independent significant prognostic factor for survival. Although elevated CEA levels were an ominous prognostic finding, the 5-year survival was still $22.7 \%$ after pulmonary resection. Thus, we do not exclude patients with an elevated CEA level from surgery.

Approximately 50\% of patients (84/167) presented with a solitary metastasis and demonstrated a significantly better survival compared with patients with multiple nodules. In addition, patients with up to 4 metastases (139/167) demonstrated a better survival than patients with more than 4 lesions.

At present, it seems that a patient with a solitary metastasis potentially benefits more from pulmonary resection than a patient with multiple pulmonary lesions. ${ }^{6,9}$ By assigning patients to prognostic categories, including the parameters of number of metastases and lymph node involvement, we could take into account all of the 167 patients. This grouping system may be helpful in selecting patients for resection of pulmonary metastases (Figure 5).

The findings of most series ${ }^{8,9,11,18}$ concur with ours: The DFI does not affect 5-year survival; however, this is in contrast with the results of Briester and colleagues. ${ }^{28}$
TABLE 3. Number of patients undergoing pulmonary metastasectomy within each year after resection of colorectal cancer

\begin{tabular}{cc}
\hline Disease-free interval (y) & Patients (No.) \\
\hline $0-1$ & 36 \\
$1-2$ & 37 \\
$2-3$ & 28 \\
$3-4$ & 23 \\
$4-5$ & 17 \\
$5-6$ & 4 \\
$6-7$ & 8 \\
$7-8$ & 2 \\
$8-9$ & 2 \\
$9-10$ & 6 \\
$10-15$ & 2 \\
$15-28$ & 2 \\
\hline
\end{tabular}

TABLE 4. Relationship of individual variables to death (Cox proportional hazards method)

\begin{tabular}{lccc}
\hline Variable & $\boldsymbol{P}$ value & Hazard ratio & $\begin{array}{c}\mathbf{9 5 \%} \text { confidence } \\
\text { limit }\end{array}$ \\
\hline $\begin{array}{l}\text { Lymph node involvement } \\
\text { Negative }\end{array}$ & .042 & & $(1.11-3.91)$ \\
$\begin{array}{l}\text { Positive } \\
\text { Number of pulmonary } \\
\quad \text { metastases }\end{array}$ & .059 & 2.09 & \\
$\quad \begin{array}{l}\text { Single } \\
\text { Multiple }\end{array}$ & & 1 & $(0.96-2.81)$ \\
$\begin{array}{l}\text { Prethoracotomy serum } \\
\text { CEA }\end{array}$ & .037 & 1.64 & \\
$\quad<5.0 \mathrm{ng} / \mathrm{mL}$ & & & \\
$>5.0 \mathrm{ng} / \mathrm{mL}$ & & 1 & \\
\hline
\end{tabular}

At the time of the diagnosis of lung metastases, other metastases, for example, hepatic lesions, are frequently found. As many as $35 \%$ of patients presented with hepatic metastases at the time of exploration for resection of primary colon cancer. ${ }^{29}$ Several authors reported similar results in survival analysis after pulmonary metastasectomy of patients in the presence of controlled hepatic metastases. 9,20 Similarly, we did not find a significant adverse effect on survival in patients after complete resection of hepatic metastases. Thus, we conclude that in selected patients, resection of solitary liver and lung metastases may provide longer palliation or result in resolution. ${ }^{24,26,30}$

Another interesting result from our data is the finding that 5-year survival of patients who underwent repeated thoracotomies for recurrent pulmonary metastases did not differ significantly from the survival observed in patients who had only 1 thoracotomy. This indicated that repeated metastasectomy may set the clock back. 


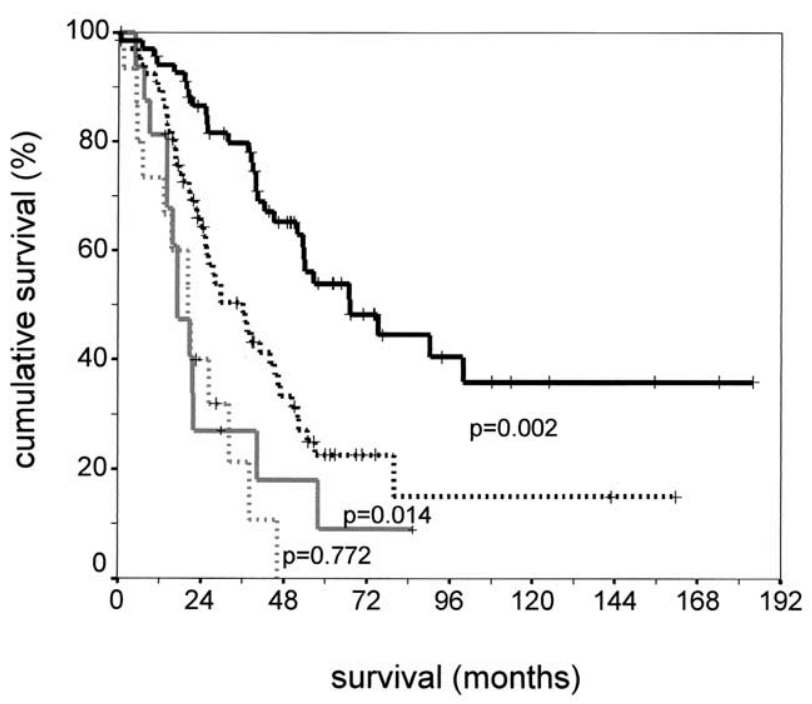

Figure 5. Kaplan-Meier survival curves according to the mode of metastases. Zero time on the abscisse represents the date of pulmonary resection (log-rank test). There was a significant survival advantage between the groups with lymph node involvement and single compared with multiple metastases $(P=.002)$ and the groups with no lymph node involvement (multiple metastases) versus lymph node involvement (single metastasis) $(\boldsymbol{P}=$ .014). Within the group with lymph node involvement and a single metastasis compared with multiple metastases, no survival advantage was detected $(P=.772)$. No lymph node involvement, 1 metastasis (black solid line); no lymph node involvement, more than 1 metastasis (black dotted line); metastatic thoracic lymph nodes, 1 metastasis (gray solid line); metastatic thoracic lymph nodes, more than one metastasis (gray dotted line).

\section{Conclusion}

Resection of pulmonary metastases of colorectal cancer is a safe and effective treatment. Low morbidity and mortality rates, contrasted with the lack of an effective systemic chemotherapy, justify the aggressive approach of surgical treatment. Once metastases have been detected, resection should not be postponed. Patients that qualify best for pulmonary resection of lung metastases from colorectal cancer have a normal serum CEA level, no evidence of mediastinal or hilar lymph node involvement, and a low number of lung metastases (eg, <4). Repeat resection for recurrent lung metastases is warranted. In selected patients, resection of solitary liver and lung metastases may provide longer palliation or may result in resolution. Lifelong surveillance is necessary to detect recurrent disease. Pulmonary resection of colorectal carcinoma is regarded as safe and effective, although the factors that determine long-term survival are not completely understood.

\section{References}

1. August DA, Ottow RT, Sugerbaker PH. Clinical prospective of human colorectal cancer metastasis. Cancer Metastasis Rev. 1984;3:303-24.
2. McCormack PM, Burt ME, Bains MS, Martini N, Rusch VW, Ginsberg RJ. Lung resection for colorectal metastases. 10-year results. Arch Surg. 1992;127:1403-6.

3. Poon MA, O'Connell MJ, Moertel CG, et al. Biochemical modulation of fluorouracil: evidence of significant improvement of survival and quality of life in patients with advanced colorectal carcinoma. J Clin Oncol. 1989;7:1407-18.

4. Divis G. Ein Beitrag zur operativen Behandlung der Lungengeschwuuilste. Acta Chir Scand. 1927;62:329-34.

5. Zanella A, Marchet A, Mainente P, Nitti D, Lise M. Resection of pulmonary metastases from colorectal carcinoma. Eur J Surg Oncol. 1997;23:424-7.

6. Okumura S, Kondo H, Tsuboi M, et al. Pulmonary resection for metastatic colorectal cancer: experiences with 159 patients. J Thorac Cardiovasc Surg. 1996;112:867-74.

7. Girard P, Ducreux M, Baldeyrou P, et al. Surgery for lung metastases from colorectal cancer: analysis of prognostic factors. J Clin Oncol. 1996;14:2047-53.

8. Baron O, Amini M, Duveau D, Despins P, Sagan CA, Michaud JL. Surgical resection of pulmonary metastases from colorectal carcinoma. Five-year survival and main prognostic factors. Eur J Cardiothorac Surg. 1996;10:347-51.

9. McAfee MK, Allen MS, Trastek VF, Ilstrup DM, Deschamps C, Pairolero PC. Colorectal lung metastases: results of surgical excision. Ann Thorac Surg. 1992;53:780-6.

10. Sauter ER, Bolton JS, Willis GW, Farr GH, Sardi A. Improved survival after pulmonary resection of metastatic colorectal carcinoma. J Surg Oncol. 1990;43:135-8.

11. Mori M, Tomoda H, Ishida T, et al. Surgical resection of pulmonary metastases from colorectal adenocarcinoma. Special reference to repeated pulmonary resections. Arch Surg. 1991;126:1297-302.

12. Headrick JR, Miller DL, Nagorney DM, et al. Surgical treatment of hepatic and pulmonary metastases from colon cancer. Ann Thorac Surg. 2001;71:975-80.

13. Abel U, Berger J, Wiebelt H. CRITLEVEL: an exploratory procedure for the evaluation of quantitative prognostic factors. Methods Inf Med. 1984;23:154-6.

14. Kaplan EL, Meier P. Nonparametric estimation from incomplete observations. J Am Stat Assoc. 1958;53:457-81.

15. Peto R, Peto J. Asymptomatically efficient rank invariant test procedures. J R Stat Soc [Ser A]. 1972;135:185-206.

16. Fisher RA, editor. Statistical methods for research workers. 14th ed. London: Olives and Boyd Limited; 1970. p. 354.

17. Cox DR. Regression models and life-table [with discussion]. J R Stat Soc Series. 1972;34:187-220.

18. Goya T, Miyazawa N, Kondo H, Tsuchiya R, Naruke T, Suemasu K. Surgical resection of pulmonary metastases from colorectal cancer. 10-year follow-up. Cancer. 1989;64:1418-21.

19. Saito Y, Omiya H, Kohno K, et al. Pulmonary metastasectomy for 165 patients with colorectal carcinoma: A prognostic assessment. J Thorac Cardiovasc Surg. 2002;124:1007-13.

20. Yano T, Hara N, Ichinose Y, Yokoyama H, Miura T, Ohta M. Results of pulmonary resection of metastatic colorectal cancer and its application. J Thorac Cardiovasc Surg. 1993;106:875-9.

21. Dowling RD, Landreneau RJ, Miller DL. Video-assisted thoracoscopic surgery for resection of lung metastases. Chest. 1998;113(1 Suppl): 2S-5S.

22. Loehe F, Kobinger S, Hatz RA, Helmberger T, Loehrs U, Fuerst H. Value of systematic mediastinal lymph node dissection during pulmonary metastasectomy. Ann Thorac Surg. 2001;72:225-9.

23. Jauch KW, Koebe HG, Piltz S, Hertlein H, Dienemann H. [Surgery of metastases - can surgery of metastases be radical?]. Zentralbl Chir. 1993;118:508-15.

24. Cahan WG, Castro EB, Hajdu SI. Therapeutic pulmonary resection of colonic carcinoma metastatic to lung. Dis Colon Rectum. 1974;17: 302-9.

25. Regnard JF, Grunenwald D, Spaggiari L, et al. Surgical treatment of hepatic and pulmonary metastases from colorectal cancers. Ann Thorac Surg. 1998;66:214-9.

26. Lehnert T, Knaebel HP, Duck M, Bulzebruck H, Herfarth C. Sequen- 
tial hepatic and pulmonary resections for metastatic colorectal cancer. Br J Surg. 1999;86:241-3.

27. Inoue M, Kotake Y, Nakagawa K, Fujiwara K, Fukuhara K, Yasumitsu T. Surgery for pulmonary metastases from colorectal carcinoma. Ann Thorac Surg. 2000;70:380-3.

28. Brister SJ, de Varennes B, Gordon PH, Sheiner NM, Pym J. Contemporary operative management of pulmonary metastases of colorectal origin. Dis Colon Rectum. 1988;31:786-92.
29. Bengmark S, Hafstrom L. The natural history of primary and secondary malignant tumors of the liver. I. The prognosis for patients with hepatic metastases from colonic and rectal carcinoma by laparotomy. Cancer. 1969;23:198-202.

30. Robinson BJ, Rice TW, Strong SA, Rybicki LA, Blackstone EH. Is resection of pulmonary and hepatic metastases warranted in patients with colorectal cancer? J Thorac Cardiovasc Surg. 1999;117:66-76.

\section{Bound volumes available to subscribers}

Bound volumes of The Journal of Thoracic and Cardiovascular Surgery are available to subscribers (only) for the 2003 issues from the Publisher, at a cost of $\$ 134.00$ for domestic, $\$ 165.85$ for Canadian, and $\$ 155.00$ for international subscribers for Vol 125 (January-June) and Vol 126 (July-December). Shipping charges are included. Each bound volume contains a subject and author index and all advertising is removed. The binding is durable buckram with the Journal name, volume number, and year stamped in gold on the spine. Payment must accompany all orders. Contact Mosby, Subscription Customer Service, 6277 Sea Harbor Dr, Orlando, FL 32887, USA; phone 800-654-2452 or 407-345-4000.

Subscriptions must be in force to qualify. Bound volumes are not available in place of a regular Journal subscription. 